\title{
Neoadjuvant Chemotherapy for Hypopharyngeal Squamous Cell Carcinoma and Personalized Medicine in Head and Neck Cancer
}

\author{
Eli Gordin, MD, FACS
}

Department of Otolaryngology - Head and Neck Surgery, University of Texas, Southwestern Medical Center, Dallas, TX

The treatment of head and neck cancer has long stood on the three pillars of surgery, chemotherapy and radiation. While the indications for and timing of administration of these modalities fluctuates, the fundamental principles of treatment have remained essentially unchanged. Therapeutic shifts have been limited to refinements in these techniques, such as minimally invasive surgery, modifications in radiation delivery, and the timing of chemotherapy administration; however, minimal improvement in survival for advanced cancers has been attained. Meanwhile, targeted therapies have shown promising results for treating cancer of the breast, colon, and lung, although head and neck cancer has enjoyed less robust success.

The study by Park and colleagues entitled A clinical trial of combination neoadjuvant chemotherapy and transoral robotic surgery in patients with T3 and T4 laryngohypopharyngeal cancer combines transoral robotic surgery (TORS) and neoadjuvant chemotherapy (NACT) as a surgical organ preservation therapy. Thirty-five patients with T3 or T4 laryngeal or hypopharyngeal squamous cell carcinoma were treated with NACT followed by TORS in cases with a 'more than partial response', with the remaining patients treated with either open surgery or definitive chemoradiotherapy (although data on these unfavorable responding patients are not presented). Threeyear disease-specific survival was $82.4 \%$, with $69.48 \%$ of patients remaining disease-free. Functional outcomes were good, with all but four patients decannulated and $83 \%$ receiving adequate nutrition by mouth.

(C) Society of Surgical Oncology 2018

First Received: 30 October 2017;

Published Online: 16 January 2018

E. Gordin, MD, FACS

e-mail: Eli.Gordin@UTSouthwestern.edu
Another recent study by Joshi et al. examined the results of NACT followed by open surgical resection of T3 and T4 laryngeal and hypopharyngeal squamous cell carcinoma. ${ }^{1}$ Thirty-five patients with 'nearly' resectable disease were administered preoperative two- or three-drug chemotherapy for two cycles before radiographic assessment for response. Twelve patients who were deemed to have 'sufficient' shrinkage underwent surgery in the form of total laryngectomy with partial or total pharyngectomy, followed by adjuvant chemoradiation or radiation alone. On pathological examination, four patients achieved complete response after NACT. Median progression-free survival was 20 months.

Induction chemotherapy (IC) has also been used prior to definitive radiotherapy for hypopharyngeal tumors. Prades et al. utilized cisplatin and 5-fluorouracil followed by radiotherapy for responses $>80 \%$, with surgery for nonresponders, compared with concurrent chemoradiation, for T3 tumors. ${ }^{2}$ Larynx preservation, local control, and distant metastasis rates were all superior with concurrent chemoradiation compared with the IC group, although 2-year survival was not statistically different.

Several challenges are faced when trying to compare these studies. First, the chemotherapy regimens used for IC/NACT are all different in terms of the drugs used and the schedule of administration, which may have a tangible impact on results. For example, Patil et al. examined unresectable oral cavity tumors treated with NACT and subsequent surgical resection if deemed amenable. A threedrug NACT regimen resulted in resectability in $66 \%$ of patients, versus $40 \%$ of patients when a two-drug regimen was employed. The use of three agents was the only factor noted to predict resectability following NACT. ${ }^{3}$

Second is the methodology employed when assessing the effect of NACT. The Response Evaluation Criteria In Solid Tumors (RECIST) is commonly used for this purpose, however concern has been raised over this system's 
reliance on the largest tumor dimension measured on imaging, which may be more appropriate in other areas of the body where spherical tumors are more common, compared with three-dimensionally complex tumor shapes often encountered in the head and neck. ${ }^{4}$ Additionally, these studies all define partial response to NACT differently in terms of percentage of tumor volume.

Third is the question of the resection margin after NACT. The utilization of pretreatment margins for postNACT resection in the head and neck is not universally adopted. Sahu and collaborators describe their experience with NACT in the treatment of T4 buccal mucosal cancers with a matched-pair analysis of 215 patients undergoing primary resection compared with 215 patients receiving NACT followed by surgery for cases deemed high risk for positive margins. ${ }^{5}$ Margins were dictated by the postNACT tumor size. There were three cases of positive margins in the group undergoing primary surgery and no cases in the NACT group. Likewise, in the study by Patil et al. mentioned above, resection was also based on tumor size after NACT. Margins $>5 \mathrm{~mm}$ were noted on pathological review of 269 of 294 specimens.

On the other side of the spectrum, Sadeghi and co-authors describe their rigorous technique for pretreatment documentation of oropharyngeal tumor volume by video recording, photography, magnetic resonance imaging (MRI), and mucosal tattooing. ${ }^{6}$ Three-drug NACT was administered to most patients, with transoral surgery offered to responders after three cycles, based on tumor margins at initial presentation. Seventeen patients were included, one of whom had positive margins.

In the present study by Park et al., the authors state that the resection of shrunken tumors included the entire anatomic compartment involved, although it is unclear if this refers to the mucosal subsite alone, or also to the deep preoperative extent of the tumor into the neck. It is interesting that $29 \%$ of patients in this article had positive surgical margins, considering the care taken in excising the pretreatment tumor volume. It would be interesting to know if residual tumor was usually seen on the deep resection surface or at the mucosal margin.

Additionally, the question is raised whether favorable responses to NACT signify tumors that are more likely to be cured regardless of the modality employed, considering the relatively successful outcomes despite a high rate of positive margins. This possibility is reflected in the study by Ceruse and co-authors, where triple-drug IC was employed prior to chemoradiation or transoral surgery in cases of more than a 50\% response, with non-responders receiving open surgery. ${ }^{7}$ The only variable correlating with 3 -year overall survival and event-free survival was the initial response to IC.

Clearly there are challenges in evaluating the role of NACT in the treatment of hypopharyngeal cancer. There is evidence that it may be helpful for some patients, although studies are quite disparate. Ultimately, this study and others like it attempt to stratify patients, based on an initial treatment response, to identify individuals who can be successfully treated less aggressively, thereby delivering more personalized and effective cancer treatment that maximally preserves quality of life.

Head and neck cancer encompasses a biologically complex group of tumors that has proven somewhat more elusive than other forms of malignancy. Studies like these are important to progress the field, while further developments are made in elucidating molecular markers of tumor behavior and targeted therapeutics that will further enhance our ability to provide the optimal treatment for each individual patient.

FUNDING No sources of funding were used to assist in the preparation of this editorial.

DISCLOSURES Eli Gordin has no conflicts of interest to declare.

\section{REFERENCES}

1. Joshi P, Joshi A, Norohna V, et al. Role of neoadjuvant chemotherapy in advanced carcinoma of the hypopharynx and larynx. South Asian J Cancer. 2017;6(1):15-19.

2. Prades JM, Lallemant B, Garrel R, et al. Randomized phase III trial comparing induction chemotherapy followed by radiotherapy to concomitant chemoradiotherapy for laryngeal preservation in T3M0 pyriform sinus carcinoma. Acta Oto-Laryngol. 2010;130:150-55.

3. Patil VM, Prabhash K, Noronha V. Neoadjuvant chemotherapy followed by surgery in very locally advanced technically unresectable oral cavity cancers. Oral Oncol. 2014;50(10):1000-4.

4. Patil V, Joshi A, Krishna VM. Is there a limitation of RECIST criteria in prediction of pathological response, in head and neck cancers, to postinduction chemotherapy? ISRN Oncol. 11:2013:259154.

5. Sahu P, Patil VM, Joshi A, et al. Neoadjuvant chemotherapy and surgical margin in technically unresectable buccal mucosa cancers. Oral Oncol. 2015;51(12):e91-e92.

6. Sadeghi N, Li N, Taheri R, et al. Neoadjuvant chemotherapy and transoral surgery as a definitive treatment for oropharyngeal cancer: a feasible novel approach. Head Neck. 2016;38(12):1837-46.

7. Ceruse P, Cosmidis A, Belot A. A pyriform sinus cancer organ preservation strategy comprising induction chemotherapy with docetaxel, cisplatin, and 5-fluorouracil, followed by potentiated radiotherapy: a multicenter, retrospective study. Anti Cancer Drugs. 2014;25(8):970-75. 